Konrad-Zuse-Zentrum für Informationstechnik Berlin

Takustr. 7, D-14195 Berlin - Dahlem

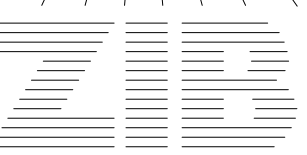

Rudolf Beck

Ralf Hiptmair*

\title{
Multilevel Solution of the Time-Harmonic Maxwell's Equations Based on Edge Elements
}

$*$

Institut für Mathematik

Universität Augsburg

D-86135 Augsburg

Preprint SC 96-51 (December 1996) 



\title{
Multilevel Solution of the Time-Harmonic Maxwell's Equations Based on Edge Elements
}

\author{
Rudolf Beck Ralf Hiptmair
}

\begin{abstract}
A widely used approach for the computation of time-harmonic electromagnetic fields is based on the well-known double-curl equation for either $\vec{E}$ or $\vec{H}$. An appealing choice for finite element discretizations are edge elements, the lowest order variant of a $H(\mathbf{c u r l})$-conforming family of finite elements.

However, the large nullspace of the curl-operator gives rise to serious difficulties. It comprises a considerable part of all spectral modes on the finite element grid, tending to pollute the solution with non-physical contributions and crippling standard multilevel solvers.

We tackle these problems by an adaptive multilevel algorithm. After every standard V-cycle with respect to the canonical basis of edge elements, the nonphysical contributions are removed by a projection step. It requires the solution of Poisson's equation, augmented by certain boundary terms, in the nullspace, which can be carried out efficiently by an inner multilevel iteration. The whole scheme yields convergence rates independent of the refinement level of the mesh.

Furthermore, a simple criterion for meshes is derived which can resolve all field modes corresponding to negative eigenvalues. This requirement is essential to guarantee both stability and efficiency of an iterative multilevel solver for indefinite systems. For controlling adaptive mesh refinement we have devised an a posteriori error indicator based on stress recovery.

Numerical experiments demonstrate the efficiency of the method for the simulation of waveguides.
\end{abstract}

Key words. Maxwell's equations, edge elements, Nédélec elements, scattering problems, waveguide computations

AMS(MOS) subject classifications. 65P05 65N30 65N55 35Q60 


\section{Introduction}

The past two decades have seen numerical simulation becoming an indispensable tool for the analysis and the design of technical devices in electrical engineering. In the high-frequency domain passive elements (e.g. waveguides and transmission lines) are typically characterized by a set of frequency dependent parameters like scattering coefficients. They can be obtained from the solution of partial differential equations which have their origin in Maxwell's equations. If the structures have complex geometries, the only feasible option is the numerical calculation of approximate solutions. The finite element method has emerged as a powerful tool for this purpose.

Plenty of finite element techniques for electromagnetic fields have been investigated (see e.g. [24, 36]). In particular, in recent years edge elements, sometimes also called Whitney forms of the lowest order, have gained in popularity as a finite element scheme for the calculation of electric fields. Contrary to nodal elements, they only enforce the continuity of the tangential field components, whereas the proper jump conditions of the normal components hold only in a weak sense. Such $H$ (curl)-conforming finite element spaces were introduced in an abstract and comprehensive manner by Nédélec [32], but it took some time until their distinct advantages in electromagnetic field computations were fully appreciated $[6,10,16]$.

As for nodal elements, there is no obvious way how to handle fields at re-entrant boundaries like at the sharp edges of ideal conductors. The same is true for similarly shaped interfaces between regions with an abrupt change of the material properties. At such points no unique surface normal exists and there is no point in enforcing all continuity relations simultaneously; a problem, which is avoided gracefully in the case of Whitney forms.

Furthermore, edge elements are well-suited to avoid the pollution of a numerical solution by spurious modes [11]. These unwanted contributions arise from the nullspace of the curl-operator in Maxwell's equations and can be suppressed in a weak sense by a proper variational principle. However, as this nullspace is rather large, it may lead to severely ill-conditioned matrices of the linear systems of equations. So conventional iterative solvers, which are indispensable in the case of large-scale three-dimensional problems, exhibit poor convergence properties.

Thus fast iterative solvers are badly needed to curb soaring computational costs on fine meshes. For a wide range of boundary value problems for partial differential equations multilevel/multigrid methods are now employed as fast iterative solvers of the discrete problems. However, these techniques are only beginning to be adapted for the computation of electromagnetic fields (cf. [31]). They can not only boost the efficiency of a simulation code by orders of magnitude, but also neatly mesh with adaptive strategies based on a-posteriori error estimation and local mesh refinement: adaptive refinement creates the very hierarchy of meshes the multilevel solver operates on. The importance of locally refined meshes for simulations of various electromagnetic phenomena has been realized by several authors $[33,18,26]$. This offers another incentive to look for an appropriate multilevel solver.

This paper introduces a new multigrid method for the scattering problems in the 
frequency domain. It is presented along with a suitable strategy for adaptive refinement of the finite element mesh based on an error indicator and unstructured tetrahedral meshes. The design of the multigrid algorithm heavily exploits the exceptional features of edge elements, in particular the existence of discrete potentials in another finite element space. They make it possible to adjust the generic linear multigrid algorithm in order to cope with the inherent lack of ellipticity of the problems under consideration.

The resulting algorithms work well both in lossy and lossless media and on locally refined grids. Heavy local refinement turns out to be essential in regions with singular fields, like at re-entrant corners, and near steep jumps of material coefficients. Here again we encounter the typical situations where edge elements exhibit their specific virtues.

The outline of the paper is a follows: in the next section we present the general setting and introduce the weak (variational) formulation of the boundary value problem. In the third section we review the construction and some properties of edge elements. In the forth section we give a detailed description of the new multigrid algorithm and explain the rationale behind its various components. The fifth section is devoted to the construction of a local error indicator based on averaging techniques. The final section examines the performance of the adaptive multilevel scheme when applied to simulate a waveguide taper device.

\section{The Time-Harmonic Maxwell's Equations}

Since the technical devices whose simulation is the goal of our research are usually characterized in the frequency domain, we restrict our simulations to time-harmonic electromagnetic fields varying with an angular frequency $\omega>0$. In this case we have for all times $t \in \mathbb{R}$ the representation

$$
\begin{aligned}
\mathcal{E}(\boldsymbol{x}, t) & =\boldsymbol{E}(\boldsymbol{x}) e^{i \omega t} \\
\mathcal{H}(\boldsymbol{x}, t) & =\boldsymbol{H}(\boldsymbol{x}) e^{i \omega t}
\end{aligned}
$$

for the electric and magnetic field $\mathcal{E}$ and $\mathcal{H}$, respectively. $\boldsymbol{E}(\boldsymbol{x})$ and $\boldsymbol{H}(\boldsymbol{x})$ are complex amplitudes defined on the simply connected bounded domain $\Omega \subset \mathbb{R}^{3}$, which designates the domain of computation. We confine ourselves to linear isotropic media without free charges. In this case Maxwell's equations state that on $\Omega$

$$
\begin{aligned}
\operatorname{curl} \boldsymbol{H} & =i \omega \epsilon \boldsymbol{E} \\
\operatorname{curl} \boldsymbol{E} & =-i \omega \mu \boldsymbol{H} .
\end{aligned}
$$

Here $\mu$ is the permeability and $\epsilon$ represents a complex dielectric constant, related to the generic dielectric constant $\epsilon^{\prime}$ and the conductivity $\sigma$ by

$$
\epsilon=\epsilon^{\prime}-i \sigma / \omega
$$

This assumption is valid in regions where the current density $\boldsymbol{j}$ can be obtained from Ohm's law by $\boldsymbol{j}=\sigma \boldsymbol{E}$. Both $\epsilon$ and $\mu$ are uniformly positive functions in $L^{\infty}(\Omega)$, where $\epsilon$ may have large jumps at material interfaces. 
In the following we shall consider two types of boundary conditions. The first is imposed on the surface part $\Gamma_{D}$, formed by a perfect conductor. This leads to a Dirichlet boundary condition enforcing zero tangential components of the electric field:

$$
\boldsymbol{n} \times \boldsymbol{E}=0 \quad \text { on } \Gamma_{D} .
$$

Here $\boldsymbol{n}$ denotes outer unit normal of the surface. Furthermore, on the remaining part $\Gamma_{C}$ of the boundary $\Gamma$ we assume that absorbing Silver-Müller boundary conditions [2] hold. They are expressed by the Cauchy type boundary conditions

$$
\boldsymbol{n} \times\left(\frac{1}{\mu} \operatorname{curl} \boldsymbol{E}\right)+\boldsymbol{n} \times(\alpha \boldsymbol{n} \times \boldsymbol{E})=\zeta \boldsymbol{E}^{i n c} \quad \text { on } \Gamma_{C} .
$$

Here $\alpha$ and $\zeta$ are scalar functions, $\boldsymbol{E}^{i n c}$ is a prescribed tangential field and plays the role of an excitation, e.g. by an incoming wave. These boundary conditions are suitable for artificial boundaries in scattering problems for waveguide structures [24], since they are specifically designed to let outgoing waves pass through unaffected.

Formally, by multiplying (2) with with $1 / \mu$, taking the curl of this equation and substituting into (1) we get

$$
\operatorname{curl} \frac{1}{\mu} \operatorname{curl} \boldsymbol{E}-\omega^{2} \epsilon \boldsymbol{E}=0 .
$$

To cast (5) together with (4) into variational form we recall the fundamental principle that the solution must be a stationary point of the Lagrangian. This means that we have to look for a saddle point of the functional

$$
F(\boldsymbol{E})=\left(\frac{1}{\mu} \operatorname{curl} \boldsymbol{E}, \operatorname{curl} \boldsymbol{E}\right)_{0}-\omega^{2}(\epsilon \boldsymbol{E}, \boldsymbol{E})_{0}+[\alpha \boldsymbol{n} \times \boldsymbol{E}, \boldsymbol{n} \times \boldsymbol{E}]_{\Gamma_{C}}-2\left[\zeta \boldsymbol{E}, \boldsymbol{E}^{i n c}\right]_{\Gamma_{C}} .
$$

To simplify the notation, we introduced the following abbreviations for inner products and boundary integrals:

$$
\begin{aligned}
(\boldsymbol{u}, \boldsymbol{v})_{0} & =\int_{\Omega} \boldsymbol{u} \boldsymbol{v} d \Omega \\
{[\boldsymbol{u}, \boldsymbol{v}]_{\Gamma} } & =\int_{\Gamma} \boldsymbol{u} \boldsymbol{v} d \Gamma
\end{aligned}
$$

The appropriate domain of definition of the functional (6), which additionally accommodates the Dirichlet boundary conditions, is given by

$$
\boldsymbol{H}_{\Gamma_{D}}(\operatorname{curl} ; \Omega):=\left\{\boldsymbol{w} \in\left(L^{2}(\Omega)\right)^{3} ; \operatorname{curl} \boldsymbol{w} \in\left(L^{2}(\Omega)\right)^{3}, \boldsymbol{w} \times \boldsymbol{n}=0 \text { on } \Gamma_{D}\right\}
$$

The field $\boldsymbol{E}$ has to be determined such that the first variation of (6) vanishes:

Seek $\boldsymbol{E} \in \boldsymbol{H}_{\Gamma_{D}}(\mathbf{c u r l} ; \Omega)$ such that for all $\boldsymbol{w} \in \boldsymbol{H}_{\Gamma_{D}}(\mathbf{c u r l} ; \Omega)$

$$
\left(\frac{1}{\mu} \operatorname{curl} \boldsymbol{E}, \operatorname{curl} \boldsymbol{w}\right)_{0}-\omega^{2}(\epsilon \boldsymbol{E}, \boldsymbol{w})_{0}+[\alpha \boldsymbol{E} \times \boldsymbol{n}, \boldsymbol{w} \times \boldsymbol{n}]_{\Gamma_{C}}=\left[\zeta \boldsymbol{E}^{i n c}, \boldsymbol{w}\right]_{\Gamma_{C}} .
$$


For $\sigma \neq 0$ the bilinear form $a: \boldsymbol{H}_{\Gamma_{D}}(\mathbf{c u r l} ; \Omega) \times \boldsymbol{H}_{\Gamma_{D}}(\mathbf{c u r l} ; \Omega) \mapsto \mathbb{R}$ occurring in $(7)$ is coercive. The challenging case is $\sigma=0$, where (7) may not have any solutions at all for certain values of $\omega$. In this presentation we focus on the case $\sigma=0$ but we want to stress that the numerical schemes work for nonvanishing $\sigma$ as well. For a detailed discussion concerning existence and uniqueness of solutions we refer to $[15,27]$.

In the sequel we require that (7) has a unique solution. Nevertheless, the operator $A$ associated with $a$ can have several negative eigenvalues if $\omega$ is large. In this case $a$ is still symmetric, but indefinite.

\section{The Finite Element Spaces}

We aim at a conforming finite element discretization of (7). To this end we pick a finite dimensional subspace of $\boldsymbol{H}_{\Gamma_{D}}(\mathbf{c u r l} ; \Omega)$ that consists of piecewise polynomials with respect to a triangulation $\mathcal{T}_{h}$ of $\Omega$. Such subspaces are provided by Nédélec's curlconforming finite elements [32], which are available for both simplicial and hexahedral triangulations. On tetrahedra Nédélec's lowest order elements coincide with so-called Whitney 1-forms [39, 10].

To be able to handle complex geometries and on behalf of a-posteriori grid adaption, we preferred tetrahedral meshes for our computations. Numerical evidence also hints at superior accuracy compared to hexahedral meshes [24]. For a tetrahedron $T$ the local lowest order Nédélec space is given by

$$
\mathcal{N} \mathcal{D}(T):=\left\{\boldsymbol{x} \mapsto \boldsymbol{a}+\boldsymbol{b} \times \boldsymbol{x} ; \boldsymbol{x} \in T, \boldsymbol{a}, \boldsymbol{b} \in \mathbb{R}^{3}\right\} .
$$

Suitable local degrees of freedom arise from path integrals along the edges of an element $T$ and can be expressed by the functionals

$$
\boldsymbol{w}_{h} \mapsto \int_{e}\left\langle\boldsymbol{w}_{h}, \boldsymbol{t}\right\rangle d s, \quad e \text { is edge of } T, \quad \boldsymbol{w}_{h} \in \mathcal{N} \mathcal{D}(T) .
$$

Therefore these elements have been dubbed "edge-elements". Unisolvence of these degrees of freedom has been established in [32]. They also ensure that the global finite element space $\mathcal{N} \mathcal{D}\left(\mathcal{T}_{h}\right)$ is contained in $\boldsymbol{H}(\mathbf{c u r l} ; \Omega)$. The corresponding canonical basis functions are locally supported and attached to the edges of the mesh $\mathcal{T}_{h}$. If $\boldsymbol{w}_{e}$ denotes the basis function belonging to the edge $e$, a straightforward computation establishes the simple representation (see e.g. [35])

$$
\boldsymbol{w}_{e}=\lambda_{i} \operatorname{grad} \lambda_{j}-\lambda_{j} \operatorname{grad} \lambda_{i}
$$

on a tetrahedron adjacent to $e$. There $\lambda_{i}$ and $\lambda_{j}$ stand for the barycentric coordinate functions associated with both endpoints of $e$. Thanks to (9) the entries of the stiffness matrix related to the bilinear form $a(\cdot, \cdot)$ can be computed efficiently. We end up with a linear system of equations

$$
\mathbb{A} \boldsymbol{u}=\boldsymbol{b}
$$

for the complex coefficient vector $\boldsymbol{u}$ of the discrete electric field $\boldsymbol{E}_{h} \in \mathcal{N} \mathcal{D}\left(\mathcal{T}_{h}\right)$ with respect to the finite element basis $\left\{\boldsymbol{w}_{e} ; e\right.$ edge of $\left.\mathcal{T}_{h}\right\}$. The dimension of this system of linear equations equals the number of edges in $\Omega \cup \Gamma_{C}$. 
Higher order $\boldsymbol{H}_{\Gamma_{D}}(\mathbf{c u r l} ; \Omega)$-conforming elements of this type are also available [35]. Approximation properties of edge elements are studied in [30]. Roughly speaking, in the lowest order case they display first order accuracy with respect to the $\boldsymbol{H}_{\Gamma_{D}}(\operatorname{curl} ; \Omega)$-norm.

There are several compelling reasons to prefer edge elements to other finite element discretizations of $\boldsymbol{H}_{\Gamma_{D}}(\mathbf{c u r l} ; \Omega)$ (cf. [12]): by virtue of their construction edge elements guarantee the continuity of tangential components across inter-element boundaries. Thus they adequately reflect continuity properties of the electric field. This is ultimately due to their close relationship to 1 -forms [21], the kind of differential forms that can be used to describe the electric field in an abstract mathematical formulation of Maxwell's equations [4].

Secondly, the use of edge elements greatly facilitates the treatment of Dirichlet boundary conditions; degrees of freedom on $\Gamma_{D}$ are simply set to zero. We write $\mathcal{N} \mathcal{D}_{\Gamma_{D}}\left(\mathcal{T}_{h}\right)$ for the resulting finite element space. Since tangential components of the discrete fields on any boundary faces are readily available, the Cauchy-conditions are as easily implemented.

Thirdly, an outstanding feature of edge elements is the fact that irrotational vector fields in $\mathcal{N} \mathcal{D}\left(\mathcal{T}_{h}\right)$ posses easily accessible discrete potentials, in stark contrast to alternative finite element approximations. This property turns out to be crucial for the multilevel scheme:

Theorem 1 Let $\mathcal{S}_{\Gamma_{D}}\left(\mathcal{T}_{h}\right)$ be the space of continuous, piecewise linear finite element functions over $\mathcal{T}_{h}$ that vanish on $\Gamma_{D}$. Then for any $\boldsymbol{w}_{h} \in \mathcal{N} \mathcal{D}_{\Gamma_{D}}\left(\mathcal{T}_{h}\right)$ with curl $\boldsymbol{w}_{h}=0$ we can find a $\phi_{h} \in \mathcal{S}_{\Gamma_{D}}\left(\mathcal{T}_{h}\right)$ such that $\boldsymbol{w}_{h}=\operatorname{grad} \phi_{h}$.

For the proof of this theorem we refer to [21].

As a consequence, the $L^{2}(\Omega)$-orthogonal discrete Helmholtz decomposition

$$
\mathcal{N} \mathcal{D}_{\Gamma_{D}}\left(\mathcal{T}_{h}\right)=\operatorname{grad} \mathcal{S}_{\Gamma_{D}}\left(\mathcal{T}_{h}\right) \oplus \mathcal{N} \mathcal{D}_{\Gamma_{D}}^{\perp}\left(\mathcal{T}_{h}\right)
$$

with the space of discrete weakly divergence free vector fields

$$
\mathcal{N} \mathcal{D}_{\Gamma_{D}}^{\perp}\left(\mathcal{T}_{h}\right):=\left\{\boldsymbol{w}_{h} \in \mathcal{N} \mathcal{D}_{\Gamma_{D}}\left(\mathcal{T}_{h}\right) ;\left(\boldsymbol{w}_{h}, \operatorname{grad} \phi_{h}\right)_{0}=0 \quad \forall \phi_{h} \in \mathcal{S}_{\Gamma_{D}}\left(\mathcal{T}_{h}\right)\right\}
$$

is no longer a mere theoretical tool, but gains relevance for the design of practical algorithms. For instance, Th. 1 offers a convenient way to enforce the (weak) divergence free condition for discrete vector fields.

If, for the moment, we neglect terms contributed by the Cauchy type boundary conditions (4), then for any solution $\boldsymbol{E}_{h}$ of the discretized problem we immediately have $\left(\epsilon \boldsymbol{E}_{h}, \operatorname{grad} \phi_{h}\right)_{0}=0$ for all $\phi_{h} \in \mathcal{S}_{\Gamma_{D}}\left(\mathcal{T}_{h}\right)$. In case $\boldsymbol{E}_{h}$ lacks this property it is possible to determine a curl-free correction grad $\psi_{h}$ by an auxiliary problem:

Seek $\psi_{h} \in \mathcal{S}_{\Gamma_{D}}\left(\mathcal{T}_{h}\right)$ such that

$$
\left(\epsilon \operatorname{grad} \psi_{h}, \operatorname{grad} \phi_{h}\right)_{0}=-\left(\epsilon \boldsymbol{E}_{h}, \operatorname{grad} \phi_{h}\right)_{0} \quad \forall \phi_{h} \in \mathcal{S}_{\Gamma_{D}}\left(\mathcal{T}_{h}\right)
$$


The variational problem (11) is a standard second order elliptic problem discretized by means of linear finite elements. Then the corrected vector field $\boldsymbol{E}_{h}+\operatorname{grad} \psi_{h}$ is obviously weakly solenoidal.

The situation gets considerably more intricate if we consider the complete functional (7), where also the Cauchy boundary conditions are taken into account. Then $\boldsymbol{E}_{h}$ is sought in the affine subspace of vector fields $\boldsymbol{\xi}_{h} \in \mathcal{N} \mathcal{D}_{\Gamma_{D}}\left(\mathcal{T}_{h}\right)$ satisfying for all $\phi_{h} \in \mathcal{S}_{\Gamma_{D}}\left(\mathcal{T}_{h}\right)$

$$
-\omega^{2}\left(\epsilon \boldsymbol{\xi}_{h}, \operatorname{grad} \phi_{h}\right)_{0}+\left[\alpha \boldsymbol{\xi}_{h} \times \boldsymbol{n}, \operatorname{grad} \phi_{h} \times \boldsymbol{n}\right]_{\Gamma_{C}}=\left[\zeta \boldsymbol{E}^{i n c}, \operatorname{grad} \phi_{h}\right]_{\Gamma_{C}} .
$$

Finding a curl-free correction of $\boldsymbol{E}_{h}$ that carries it into this space amounts to solving the following variational problem, in analogy to (11):

Seek $\psi_{h} \in \mathcal{S}_{\Gamma_{D}}\left(\mathcal{T}_{h}\right)$ such that for all $\phi_{h} \in \mathcal{S}_{\Gamma_{D}}\left(\mathcal{T}_{h}\right)$

$$
\begin{aligned}
& \left(\epsilon \operatorname{grad} \psi_{h}, \operatorname{grad} \phi_{h}\right)_{0}-\left[\frac{\alpha}{\omega^{2}} \boldsymbol{n} \times \operatorname{grad} \psi_{h}, \boldsymbol{n} \times \operatorname{grad} \phi_{h}\right]_{\Gamma_{C}}= \\
& =-\left(\epsilon \boldsymbol{E}_{h}, \operatorname{grad} \phi_{h}\right)_{0}+\left[\frac{\alpha}{\omega^{2}} \boldsymbol{n} \times \boldsymbol{E}_{h}, \boldsymbol{n} \times \operatorname{grad} \phi_{h}\right]_{\Gamma_{C}}-\left[\frac{\zeta}{\omega^{2}} \boldsymbol{E}_{h}^{i n c}, \operatorname{grad} \phi_{h}\right]_{\Gamma_{C}(13)} .
\end{aligned}
$$

This time the bilinear form associated with (13) is no longer positive definite under any circumstance. In general it is indefinite and (13) might not have any solution at all. However, for the scattering problems considered in this paper, the crucial parameter $\alpha$ in (13) is a complex number (see equations (23) and (24) in section 6). Except for the unrealistic case of extremely strong attenuation, its imaginary part is dominating and (13) can be solved efficiently with a standard multilevel algorithm. So for realistic values of $\alpha$ we did not encounter any difficulties in our simulation runs.

\section{The Multilevel Solution Procedure}

We aim to adapt the classical multigrid idea (see $[19,13])$ to the particular problem $(7)$ when discretized by means of the lowest order edge elements introduced in the previous section.

We start with a sequence of tetrahedral meshes $\left\{\mathcal{T}_{k}\right\}_{k=0}^{L}$ arising from the successive refinement of an initial coarse grid $\mathcal{T}_{0}$. Various refinement schemes have been devised $[9,3]$ that maintain shape regularity and allow local refinement. They all manage to avoid dangling nodes and rely on subdividing individual tetrahedra into a few smaller ones. Our simulations are based on the red-green refinement policy detailed in $[9,7]$. The basic action is to chop up a tetrahedron into eight small tetrahedra. It is supplemented by a few special "green" subdivisions in order to take care of dangling nodes in the case of local refinement. We can easily confirm that the edge element spaces belonging to different levels of refinement are properly nested:

$$
\mathcal{N} \mathcal{D}_{\Gamma_{D}}\left(\mathcal{T}_{0}\right) \subset \mathcal{N} \mathcal{D}_{\Gamma_{D}}\left(\mathcal{T}_{1}\right) \subset \ldots \subset \mathcal{N} \mathcal{D}_{\Gamma_{D}}\left(\mathcal{T}_{L}\right)
$$


We opt for a standard scheme and use the canonical intergrid transfer operators $P_{l-1}^{l}$ : $\mathcal{N D}_{\Gamma_{D}}\left(\mathcal{T}_{l-1}\right) \mapsto \mathcal{N} \mathcal{D}_{\Gamma_{D}}\left(\mathcal{T}_{l}\right)$ (prolongation) and $R_{l}^{l-1}: \mathcal{N} \mathcal{D}_{\Gamma_{D}}^{\prime}\left(\mathcal{T}_{l}\right) \mapsto \mathcal{N} \mathcal{D}_{\Gamma_{D}}^{\prime}\left(\mathcal{T}_{l-1}\right)$ (restriction) furnished by the embedding (14). In particular, this means that the restriction is the adjoint of the prolongation: $R_{l}^{l-1}=\left(P_{l-1}^{l}\right)^{*}$. Owing to the small supports of the edge element basis functions, both operators permit purely local evaluation. Moreover, the weights occurring in the transfer matrices only depend on the pattern of refinement of a tetrahedron and not on its shape. This permits us to compute the weights in advance for all varieties of green and red refinements.

On each level of refinement simple Gauß-Seidel steps serve as smoothers. They boil down to a successive relaxation of individual nodes. As usual in an adaptive setting [29], only those degrees of freedom are covered by relaxation on level $l$ that have been affected when $\mathcal{T}_{l-1}$ was refined into $\mathcal{T}_{l}$. This practice is often referred to as "local multigrid".

The efficacy of a multilevel approach to linear symmetric problems hinges on the proper ellipticity of the variational problem. This means that all eigenvalues should be positive and that the amplification (i.e. the eigenvalue) of an eigenfunction is proportional only to its (spatial) frequency. These conditions are obviously violated in the current setting, the multigrid approach facing two major obstacles:

1. The bilinear for $a(\cdot, \cdot)$ gives rise to an indefinite operator.

2. The problem utterly lacks ellipticity on the kernel of the curl-operator.

Considerations in the spectral domain offer a clue: in the entire space $\mathbb{R}^{3}$ we have for $\boldsymbol{w} \in \boldsymbol{H}_{\Gamma_{D}}\left(\operatorname{curl} ; \mathbb{R}^{3}\right)$ that satisfies $(\boldsymbol{w}, \operatorname{grad} \phi)_{0}=0$ for all $\phi \in H^{1}\left(\mathbb{R}^{3}\right)$

$$
(\operatorname{curl} \boldsymbol{w}, \operatorname{curl} \boldsymbol{w})_{0}=\int_{\mathbb{R}^{3}}|\boldsymbol{k}|^{2}|\widehat{\boldsymbol{w}}(\boldsymbol{k})|^{2} d \boldsymbol{k},
$$

where $\widehat{\boldsymbol{w}}$ stands for the Fourier transform of $\boldsymbol{w}$. From (15) we learn that the spectral components of weakly divergence free functions experience an amplification depending solely on the square of their frequency.

We are led to conclude that even in a bounded domain the operator $A$ still retains an elliptic character, when we restrict it to the orthogonal complement $\mathcal{N} \mathcal{D}_{\Gamma_{D}}^{\perp}\left(\mathcal{T}_{h}\right.$ ) (as defined in (10)) of the kernel of the curl-operator and ignore the space $\mathcal{M}^{-}$spanned by negative eigenmodes.

For the moment assuming homogeneous material (i.e. $\mu, \epsilon=$ const.), we infer from (15) that a plane wave $\boldsymbol{E}(\boldsymbol{x})=\exp (i \boldsymbol{k} \boldsymbol{x}) \cdot \overrightarrow{\boldsymbol{e}}_{x}$ can only coincide with a negative eigenmode if $|\boldsymbol{k}|^{2}<\omega^{2} \mu \epsilon$. In other words, its spatial wavelength must be below a critical wavelength

$$
\lambda_{C}:=\frac{2 \pi}{\omega \sqrt{\epsilon \mu}}
$$

Experience and theory (cf. [14]) teach us that a multigrid scheme for indefinite symmetric problems may perform well if all eigenfunctions corresponding to negative eigenvalues can be well represented on the coarsest grid. To solve the coarse grid problem 
we can resort to a direct method based on sparse LU-factorization, which is not sensitive to negative eigenvalues. Thus the final corrections in $\mathcal{M}^{-}$are already provided by the coarse grid solver. According to Nyquist's theorem, we need at least two sampling points per wavelength to sample a sine wave. Provided that the above heuristic considerations hold locally, we arrive at the following requirement:

The length $h_{e}$ of an edge e of the coarsest mesh $\mathcal{T}_{0}$ must satisfy

$$
h_{e} \leq \frac{\lambda_{C}}{2}=\frac{\pi}{\omega \sqrt{\epsilon^{\prime} \mu}},
$$

where the maximal values of $\epsilon$ and $\mu$ in elements adjacent to e should be taken.

For simulations of technical structures this restriction should still be feasible in most cases and need not lead to prohibitively many elements in $\mathcal{T}_{0}$. We point out that due to large variation of $\mu$ and $\epsilon$ we may end up with a grossly non-uniform initial grid.

Note that on finer meshes the diameter of supports of nodal basis functions is well below the threshold (17). Thus even a plain Gauß-Seidel smoother is impervious to the impact of negative eigenmodes, since for any $\boldsymbol{w}_{e}$ we have $a\left(\boldsymbol{w}_{e}, \boldsymbol{w}_{e}\right)>0$. This is because the dominant frequency components of $\boldsymbol{w}_{e}$ belong to the space $\mathcal{M}^{+}$spanned by eigenvectors corresponding to positive eigenvalues. In sum, the multigrid convergence should not be affected by the presence of negative eigenvalues, if the above requirement is met.

In addition, observe that for a basis function $\boldsymbol{w}_{e}$

$$
\left\|\boldsymbol{w}_{e}\right\|_{0} \leq C h\left\|\operatorname{curl} \boldsymbol{w}_{e}\right\|_{0}
$$

where $h$ is the size of the elements surrounding $e$ and $C>0$ does not depend on $h$. This estimate is quickly established through straightforward affine equivalence techniques. The inequality (18) indicates that the basis functions have a distinct oscillatory character and are sufficiently orthogonal to $\mathcal{N}(\mathbf{c u r l})$. This ensures the smoothing property of Gauß-Seidel sweeps, though they are basically unstable, i.e. the plain Gauß-Seidel method does not yield a convergent iterative method for the indefinite problem. An important consequence is that only a small number of smoothing steps makes sense, whereas many of them are detrimental. In general, we observed optimal performance for one or two pre- and postsmoothing steps.

As an alternative smoother, following [40], we could have used a relaxation based on the $(\operatorname{curl} \cdot, \operatorname{curl} \cdot)_{0}$-part of the bilinear form $a(\cdot, \cdot)$ only. This leads to a smoothing iteration that converges in the $\|$ curl $\cdot \|_{0}$-seminorm. Although this approach seems to be promising at first glance, there are serious drawbacks: First, storing another operator inflates memory requirements. Secondly, a thorough analysis reveals that the resulting multigrid cycle no longer provides a symmetric preconditioner. Yet symmetry of the preconditioning operator is essential for Krylov-methods we wish to employ for the sake of additional robustness (see below).

In the case of homogeneous materials and under some restrictions on $\Omega$ it has been shown in [22] that for uniform refinement a V-cycle multigrid method is an asymptotically optimal preconditioner for the bilinear form $(\operatorname{curl} \cdot, \mathbf{c u r l} \cdot)_{0}$ restricted to 
$\mathcal{N} \mathcal{D}^{\perp}\left(\mathcal{T}_{L}\right)$. If the negative eigenmodes are taken care of on the coarsest grid, this result will carry over to the current scheme.

Excellent convergence in the space $\mathcal{N} \mathcal{D}^{\perp}\left(\mathcal{T}_{L}\right)$ is not enough, however. Obviously the nodal basis functions $\boldsymbol{w}_{e}$ on any level $l$ do not exactly lie in $\mathcal{N} \mathcal{D}^{\perp}\left(\mathcal{T}_{l}\right)$. Invariably each smoothing step introduces some pollution in $\mathcal{N}$ (curl), hence. These unwanted contributions evidently belong to $\mathcal{M}^{-}$which makes them invisible for further smoothing steps. Eventually these spurious components might be building up and severely pollute the solution after a few $\mathrm{V}$-cycles. We cannot dismiss their impact, since the presence of a zero order term makes the smoothers susceptible to curl-free perturbations.

To weed out these $\mathcal{N}(\mathbf{c u r l})$-components a projection onto the constrained space given by (12) is required. It can be done efficiently by carrying out a few standard V-cycles for the problem (13) and using the result as a curl-free correction to the tainted approximation. In the case $\Gamma_{C}=\emptyset$ standard multigrid theory predicts rapid convergence of these iterations, virtually independent of the jumps of $\epsilon$ and the meshwidth $h$. From numerical experiments we conclude that the presence of the additional boundary term does not disrupt convergence. So we can curb curl-free errors after each V-cycle with reasonable computational effort. Please note that we entirely owe this possibility to the existence of discrete potentials for edge elements.

So we obtain an outer multilevel iteration within the space of edge elements, where each $\mathrm{V}$-cycle is followed by an inner iteration to remove the $\mathcal{N}(\mathbf{c u r l})$-components. At a first glance, this additional projection (henceforth denoted by $Q_{L}$ ) makes the nested iteration scheme structurally unsymmetric. However, if it is ensured that $Q_{L}$ is carried out with a precision exceeding the one required for the outer iteration, no substantial contributions of $\mathcal{N}(\mathbf{c u r l})$-components will appear and the symmetry of the outer multilevel scheme is preserved.

To avoid non-solenoidal pollution from the very beginning, it is essential to remove any $\mathcal{N}$ (curl)-components in the initial guess; the respective projection will be denoted by $Q_{L}^{O}$. The only difference between $Q_{L}^{O}$ and $Q_{L}$ lies in the fact that the source term defined by the incident field $\boldsymbol{E}_{h}^{i n c}$ in (13) has to be dropped in $Q_{L}$. The reason for this is that the preconditioner merely solves a defect equation, where source terms (related to the right hand side vector of the linear system) do not contribute explicitely.

As the multilevel preconditioner is symmetric it can be embedded into any suitable Krylov-subspace iteration. To enhance the robustness of the scheme, we use V-cycles as preconditioners for a conjugate residual method. This is meant to be an additional safeguard against negative eigenmodes that have eluded the coarse grid solver; it is well known that this $\mathrm{CG}$ variant can cope with symmetric indefinite systems. If only a few negative eigenvalues are present, it recovers the speed of convergence of the preconditioned CG method after a few steps (cf. [20]).

We point out that a plain preconditioned CG method does not perform worse in numerical experiments (see Sect. 5), though theory guarantees convergence only for positive definite problems. However, in the current setting the preconditioner renders negative eigenvalues virtually invisible to the Krylov-subspace iteration. Thus the convergence properties of the preconditioned CG method are preserved unscathed even in the presence of a few negative eigenvalues (cf. [37]). The basic algorithms 
Solve $\mathbb{A} \boldsymbol{u}=\boldsymbol{b}$ with initial guess $\boldsymbol{u}_{0}$ :

Remove non-solenoidal contributions: $\boldsymbol{u}_{0}=Q_{L}^{0}\left(\boldsymbol{u}_{0}\right)$

$\boldsymbol{r}_{0}=\boldsymbol{b}-\mathbb{A} \boldsymbol{u}_{0}$

$\boldsymbol{\rho}_{0}=\mathbb{C} \boldsymbol{r}_{0}$

Remove non-solenoidal contributions: $\boldsymbol{\rho}_{0}=Q_{L}\left(\boldsymbol{\rho}_{0}\right)$

$\boldsymbol{p}_{0}=\boldsymbol{\rho}_{0}$

for $k=1,2, \ldots$

$\alpha=\frac{\boldsymbol{\rho}_{k-1}^{t} \boldsymbol{r}_{k-1}}{\boldsymbol{p}_{k-1}^{t} \mathbb{A} \boldsymbol{p}_{k-1}}$

$\boldsymbol{u}_{k}=\boldsymbol{u}_{k-1}+\alpha \boldsymbol{p}_{k-1}$

$\boldsymbol{r}_{k}=\boldsymbol{r}_{k-1}-\alpha \mathbb{A} \boldsymbol{p}_{k-1}$

if $\frac{\boldsymbol{r}_{k}^{t} \boldsymbol{r}_{k}}{\boldsymbol{b}^{t} \boldsymbol{b}}<$ tol : end

$\boldsymbol{\rho}_{k}=\mathbb{C} \boldsymbol{r}_{k}$

Remove non-solenoidal contributions: $\boldsymbol{\rho}_{k}=Q_{L}\left(\boldsymbol{\rho}_{k}\right)$

$\boldsymbol{p}_{k}=\boldsymbol{\rho}_{k}+\frac{\boldsymbol{\rho}_{k-1}^{t} \boldsymbol{r}_{k-1}}{\boldsymbol{\rho}_{k}^{t} \boldsymbol{r}_{k}} \boldsymbol{p}_{k-1}$

Figure 1: Preconditioned conjugate gradient method with projection steps $Q_{L}^{O}$ and $Q_{L}$. The symbol $\mathbb{C}$ denotes the operation of the preconditioner; tol is a prescribed tolerance.

for solving $\mathbb{A} \boldsymbol{u}=\boldsymbol{b}$ by a multilevel preconditioned conjugate gradient algorithm are depicted in Figs. 1 and 2.

\section{The Adaptive Mesh Refinement}

In the presence of sharply localized phenomena the gain through local mesh refinement easily outweighs its higher computational costs (compared to uniform grids). In the case of waveguide simulation, there are typical situations, where we have to deal with near singularities of the solution, particularly in the vicinity of sharp metallic edges and corners. There the mesh should be significantly finer than in the rest of the computational domain to get a prescribed overall accuracy. To determine where and how far local refinement must be carried out, a local error monitor is needed.

Brisk research has been going on the field of local a-posteriori error estimators (cf. [38, 25, 8, 5]), but the theory has so far skirted indefinite problems. An attractive, though somewhat heuristic approach based on stress recovery was introduced by Zienkiewicz and Zhu [41]. It was investigated in more detail and extended to weighted projection schemes in [1]. These authors utilize a stress field computed from the discrete solution at points where the approximation displays superconvergence. Then this stress field is improved by a weighted $L_{2}$-projection into a suitable finite element space. The analysis in [1] is carried out for positive definite systems arising in structural mechanics, whereas our electric field computations are not based on minimization principles. So convergence results derived in [1] do not readily apply here 


$$
\begin{aligned}
\operatorname{VCycle} & \left(\boldsymbol{\rho}^{l}, \boldsymbol{r}^{l}, l\right): \\
& \text { if }(l==0): \text { solve } \mathbb{A}^{0} \boldsymbol{\rho}^{0}=\boldsymbol{r}^{0} \text { by FB-Substitution and return } \\
& \boldsymbol{\rho}^{l}=0 \\
& \boldsymbol{\rho}^{l}=\text { GS_Forward }\left(\boldsymbol{\rho}^{l}, \mathbb{A}^{l}, \boldsymbol{r}^{l}\right) \\
& \boldsymbol{r}^{l-1}=R_{l}^{l-1}\left(\boldsymbol{r}^{l}-\mathbb{A}^{l} \boldsymbol{\rho}^{l}\right) \\
& V C y c l e\left(\boldsymbol{\rho}^{l-1}, \boldsymbol{r}^{l-1}, l-1\right) \\
& \boldsymbol{\rho}^{l}=\boldsymbol{\rho}^{l}+P_{l-1}^{l}\left(\boldsymbol{\rho}^{l-1}\right) \\
& \boldsymbol{\rho}^{l}=G S \_B a c k w a r d\left(\boldsymbol{\rho}^{l}, \mathbb{A}^{l}, \boldsymbol{r}^{l}\right)
\end{aligned}
$$

Figure 2: Recursive symmetric multigrid V-cycle with Gauß-Seidel smoothing for computing $\boldsymbol{\rho}=\mathbb{C} \boldsymbol{r} . R_{l}^{l-1}$ and $P_{l-1}^{l}$ denote prolongation and restriction between levels $l$ and $l-1$.

as our functional (6) does not induce an energy norm. Hence we will call the device presented below an error indicator.

Let $\boldsymbol{E}_{h}$ denote the discrete solution for the electric field. In our case, the magnetic field $\boldsymbol{H}$ plays the part of the stress and is given by equation (2)

$$
\boldsymbol{H}_{h}=-\frac{1}{i \omega \mu} \sum_{k} \operatorname{curl} \boldsymbol{E}_{h},
$$

It is clear from taking the derivative that $\boldsymbol{H}_{h}$ does not match the order of approximation of $\boldsymbol{E}_{h}$. If $\mu$ is constant, $\boldsymbol{H}_{h}$ belongs to the space of Raviart-Thomas elements [32], which sport continuity of normal components. However, the magnetic field is a 1-form like the electric field and thus it should be approximated by edge elements. To construct such an improved approximation $\hat{\boldsymbol{H}}_{h}$, we resort to a global $L^{2}$-orthogonal projection of $\boldsymbol{H}_{h}$ onto $\mathcal{N} \mathcal{D}(\Omega)$. It can be obtained from the discrete variational problem

$$
\left(\hat{\boldsymbol{H}}_{h}-\boldsymbol{H}_{h}, \boldsymbol{w}_{h}\right)_{0}=0 \quad \forall \boldsymbol{w}_{h} \in \mathcal{N} \mathcal{D}(\Omega) .
$$

We end up with a system of linear equations corresponding to the mass matrix for edge elements. Thus its coefficient matrix is positive definite and its condition number is bounded independently of the size of the elements of the mesh. Thus the above problem can be solved efficiently by a conjugate gradient algorithm, where a dozen iterations are quite sufficient.

Now a local error indicator $\eta_{T}$ for each element $T$ in the mesh can be gained by calculating the weighted mean square deviation of both magnetic field approximations. As we are working with oscillating fields, one should take the average with respect to time by working with the complex conjugate (denoted by the asterisk ${ }^{*}$ ):

$$
\eta_{T}=\frac{1}{2} \int_{T} \mu\left(\hat{\boldsymbol{H}}_{h}-\boldsymbol{H}_{h}\right)^{*}\left(\hat{\boldsymbol{H}}_{h}-\boldsymbol{H}_{h}\right) d \Omega,
$$

Speaking in physical terms, this quantity gives the average deviation of the local magnetic energy density. 
A global indicator $\eta$ for the relative error is obtained by summing up the local deviations and dividing the result by the total magnetic energy:

$$
\eta=\frac{\frac{1}{2} \int_{\Omega} \mu\left(\hat{\boldsymbol{H}}_{h}-\boldsymbol{H}_{h}\right)^{*}\left(\hat{\boldsymbol{H}}_{h}-\boldsymbol{H}_{h}\right) d \Omega}{\frac{1}{2} \int_{\Omega} \mu \boldsymbol{H}_{h}^{*} \boldsymbol{H}_{h} d \Omega}
$$

Since the numerator in (21) is the sum of the local contributions in (20), we can use their relative size to guess at the distribution of the discretization error. In detail, we mark an element as eligible for further refinement, if its indicator $\eta_{T}$ is beyond a threshold $\Theta$ determined by the maximal local error encountered on the mesh: $\Theta=\gamma \max _{T} \eta_{T}$. Setting the parameter $\gamma$ to 0.25 is usually a satisfactory choice. To avoid any stagnation of the adaptive process, it is ensured that at least 5 per cent of all elements are marked for refinement.

Numerical experiments confirmed that the indicator is well capable of steering adaptive mesh refinement. To assess its quality as an estimator, we did some calculations for a simple test problem, where the analytical solution is available. We simulated a standing wave between two parallel metallic plates with perfectly conducting walls; the simulation area has the shape of a rectangular box. On a boundary plane perpendicular to the plates a transversal electromagnetic (TEM-) wave is excited by a Dirichlet boundary condition; the wave is reflected completely by a further metallic plate on the opposite face.

We created a regular tetrahedral mesh to discretize the simulation area. The initial grid was chosen such that the ratio between the maximal edge length and the wave length is $\frac{1}{3}$. Additionally three steps of uniform mesh refinement were carried out.

\begin{tabular}{|c|l|l|c|}
\hline Ref. Depth & $\eta$ & $\eta_{\text {true }}$ & $\eta / \eta_{\text {true }}$ \\
\hline 0 & 0.128 & 0.106 & 1.2 \\
1 & 0.0394 & 0.0216 & 1.8 \\
2 & 0.0111 & 0.00507 & 2.2 \\
3 & 0.00296 & 0.00125 & 2.4 \\
\hline
\end{tabular}

Table 1: Estimated and true errors of the test problem.

Table 1 shows the results for the different refinement levels. In the second and the third column the estimated and the true errors are printed, the last column presents the ratio of these values. The table does not indicate an asymptotic convergence for the estimated values as would be desirable; however, the agreement seems to be still acceptable to assess the quality of the numerical solution. At least the figures hint at the reliability of the error indicator; it hardly ever misses regions where there is a significant approximation error left.

Calculations with local grid refinement yielded quite a similar behavior of the error indicator. 

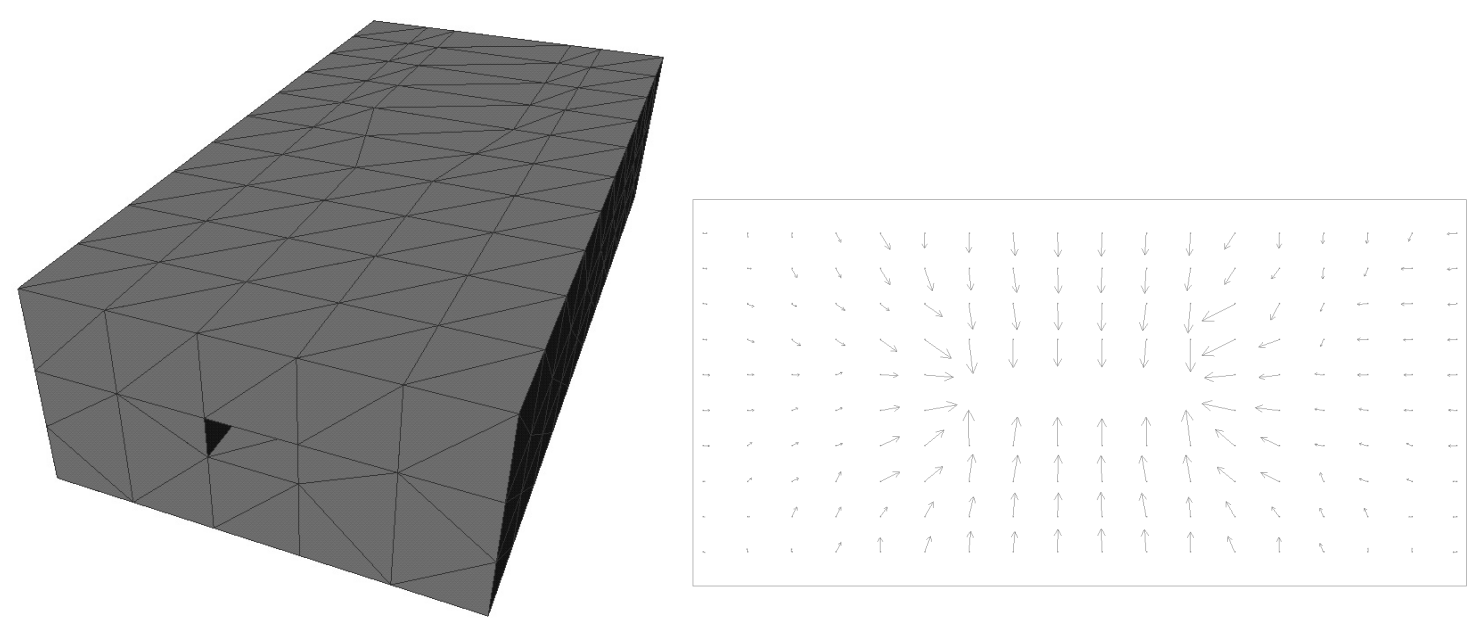

Figure 3: Initial finite element mesh of the taper structure (left) and electric field of an incident TEM-wave. The inner conductor has not been modelled.

\section{$6 \quad$ Numerical Experiments}

We calculate the electric field within a three-dimensional taper structure in a closed metallic box (see figures 3 and 4). The taper section is placed in the middle of the model and connects two microstrip lines of different width. Inner and outer conductor are formed by metallic surfaces with negligible resistance, where the tangential component of the electric field has to vanish. The inner conductor has rectangular cross sections; thus the electric field is singular at the edges and the mesh is refined adaptively in these regions.

We assume that both the input port at the front and the output port at the back of the structure propagate only TEM-modes. Thus in the immediate vicinity of each port the field may be split into an incident and a reflected TEM-wave by [28]

$$
\begin{aligned}
\boldsymbol{E}(\boldsymbol{x}) & =\boldsymbol{E}^{i n c}(\boldsymbol{x})+\boldsymbol{E}^{r e f}(\boldsymbol{x}) \\
& =\boldsymbol{E}^{i n c}(x, y) e^{-i \beta z}+\boldsymbol{E}^{r e f}(x, y) e^{i \beta z}
\end{aligned}
$$

Here the direction of propagation is aligned to the $\mathrm{z}$-axis; $\beta$ denotes the propagation constant and is given by

$$
\beta=\omega \sqrt{\epsilon \mu}
$$

For practical computations of scattering coefficients it is neccessary that the planes of both ports are placed far enough from the discontinuity region, so that all higher-order modes excited by the obstacle have decayed at the ports.

The incident field $\boldsymbol{E}^{i n c}(x, y)$ of a TEM-mode can be derived from a two-dimensional static potential for which Laplace's equation holds on the plane defining the port [34]. Of course, the numerical precision of this solution should be beyond the precision 


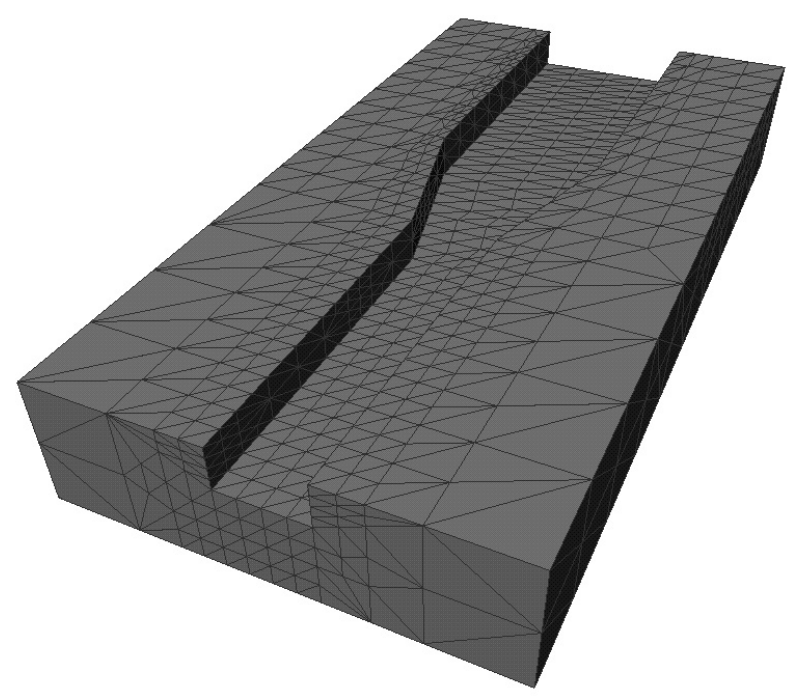

Figure 4: Mesh after the second adaptive refinement step. The elements above the inner conductor have been removed for display.

desired for the complete 3D-structure. The incident field at the front port is shown in figure 3 .

To obtain a suitable boundary condition for the ports, we apply the operator of the natural boundary condition of the functional (6) to (22) (note that on the input port the unit normal $\boldsymbol{n}$ is aligned to the negative $\mathrm{z}$-direction):

$$
\boldsymbol{n} \times\left(\frac{1}{\mu} \operatorname{curl} \boldsymbol{E}\right)=\boldsymbol{n} \times\left(-\frac{i \beta}{\mu} \boldsymbol{n} \times \boldsymbol{E}^{i n c}\right)+\boldsymbol{n} \times\left(\frac{i \beta}{\mu} \boldsymbol{n} \times \boldsymbol{E}^{r e f}\right)
$$

We may substitute $\boldsymbol{E}^{\text {ref }}$ by (22) to get

$$
\boldsymbol{n} \times\left(\frac{1}{\mu} \operatorname{curl} \boldsymbol{E}\right)=-2\left(\frac{i \beta}{\mu} \boldsymbol{E}^{i n c}\right)+\boldsymbol{n} \times\left(\frac{i \beta}{\mu} \boldsymbol{n} \times \boldsymbol{E}\right) .
$$

This equation states a Cauchy-type boundary condition like (4). On the output port $\boldsymbol{E}^{\text {inc }}$ is set to zero.

For our computations we assumed the structure to be filled with homogeneous dielectric material $\left(\epsilon_{r}=1\right.$ and $\left.\mu_{r}=1\right)$. The height of the inner conductor is $10 \mathrm{~mm}$; its width at the front port is $20 \mathrm{~mm}$ and $40 \mathrm{~mm}$ at the back. The complete structure is $200 \mathrm{~mm}$ long and $100 \mathrm{~mm}$ high, the taper section has a length of $20 \mathrm{~mm}$. We solved the problem using adaptive mesh refinement as described in section 5. On every refinement level we set the initial solution guess to zero; the requested tolerance for the outer iteration (tol in figure 1) was $10^{-6}$.

We started all computations on the initial mesh shown in figure 3, which comprises 566 degrees of freedom on the edges (dirichlet nodes are not counted). Figure 5 shows the electric field in a plane right above the inner conductor for an excitation frequency of $3 \mathrm{GHz}$ after two adaptive refinement steps. 


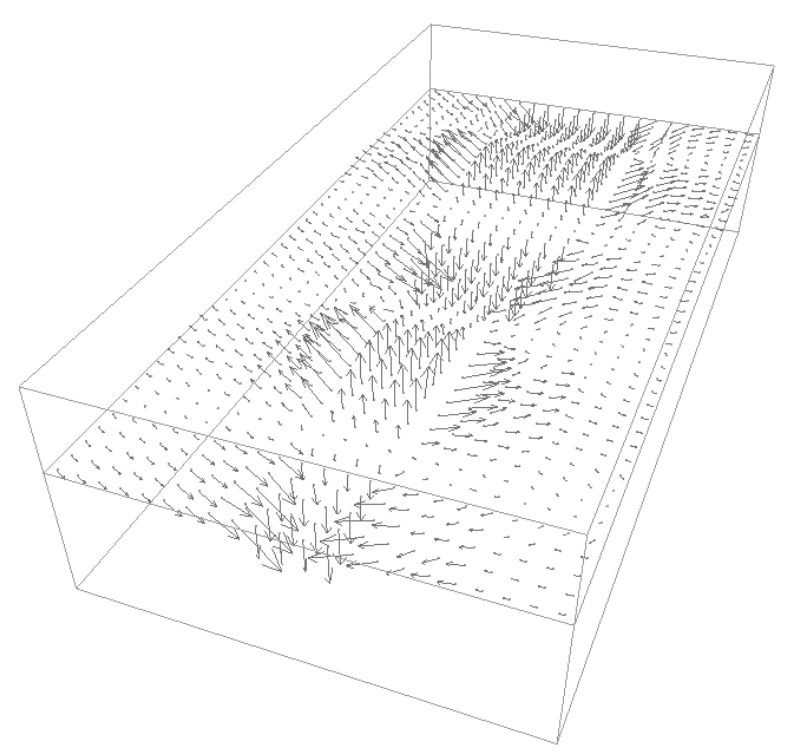

Figure 5: Electric field in a plane above the inner conductor. The excitation frequency is $3 \mathrm{GHz}$.

We applied various excitation frequencies to assess the influence of the ratio between edge length and critical wavelength

$$
r=\max \frac{h_{e}}{\lambda_{C}}
$$

with $h_{e}$ and $\lambda_{C}$ as defined in (16) and (17). As stated in (17), $r$ should be below 0.5 to ensure the convergence of the multilevel solvers.

The first run was carried out at a frequency $f=1.5 \mathrm{GHz}$, yielding $r=0.25$ on the basic mesh. So the negative eigenvalues are well resolved. To demonstrate the efficiency of the projection scheme proposed in section 4, we compare in table 2 the results obtained both with and without projection (denoted by CR-ML-P and CR-ML in the table). The smoother in the multilevel preconditioner was a local Gauß-Seidel procedure with one pre- and one post-smoothing step; for the basic Krylov-subspace solver we chose a conjugate residual algorithm.

\begin{tabular}{|c|r|rr|rr|}
\hline \multirow{2}{*}{ Ref. } & \multirow{2}{*}{ Nodes } & \multicolumn{2}{|c|}{ \#Iter } & \multicolumn{2}{|c|}{ CPU [sec] } \\
Depth & & CR-ML & CR-ML-P & CR-ML & CR-ML-P \\
\hline 1 & 2946 & 115 & 16 & 16 & 4 \\
2 & 8423 & 297 & 19 & 144 & 19 \\
3 & 25542 & 607 & 18 & 1128 & 73 \\
4 & 67549 & 1186 & 18 & 6679 & 235 \\
5 & 173264 & 2593 & 20 & 41430 & 780 \\
\hline
\end{tabular}

Table 2: Convergence history for $f=1.5 \mathrm{GHz}, r=h_{e} / \lambda_{C}=0.25$.

Table 2 shows the number of nodes created in each refinement step (the refinement 
depth in the mesh increased with every step for all the examples presented here). In the remaining colums the iteration counts and processor (CPU) times for the solvers are given. The results demonstrate that the convergence rate of the multilevel solver without projection deteriorates with increasing refinement depth. The iteration count roughly increases by a factor 2 , indicating that the smoothing operations for levels $>1$ do not speed up the convergence. On the contrary, the additional projection schemes yields a convergence rate which is independent of the refinement depth. Although it requires about 10 inner multilevel iterations for each outer iteration, this effort definitely pays off.

To study the behaviour of an alternative basic solver, we replaced the conjugate residual algorithm (CR) with a conjugate gradient iteration (CG). Furthermore, we also used the multilevel algorithm as a stand-alone solver, but with three pre-smoothing steps, which improved the convergence significantly. In all cases the projection scheme was employed. Both alternatives worked well; the results are shown in table 3. As a conjugate gradient algorithm requires fewer vector updates than a CR-iteration, it renders a better performance with regard to the CPU-times.

\begin{tabular}{|c|r|rrr|rrr|}
\hline \multirow{2}{*}{$\begin{array}{c}\text { Ref. } \\
\text { Depth }\end{array}$} & \multirow{2}{*}{ Nodes } & \multicolumn{3}{|c|}{ \#Iter } & \multicolumn{3}{|c|}{ CPU [sec] } \\
& & CR-ML-P & CG-ML-P & ML-P & CR-ML-P & CG-ML-P & ML-P \\
\hline 1 & 2946 & 16 & 16 & 14 & 4 & 4 & 5 \\
2 & 8423 & 19 & 18 & 17 & 19 & 17 & 22 \\
3 & 25542 & 18 & 18 & 21 & 73 & 71 & 111 \\
4 & 67549 & 18 & 18 & 22 & 235 & 223 & 403 \\
5 & 173264 & 20 & 19 & 23 & 780 & 747 & 1330 \\
\hline
\end{tabular}

Table 3: Convergence history for $f=1.5 \mathrm{GHz}, r=0.25$.

Setting the frequency to $3 \mathrm{GHz}$ we reach a critical region where the coarse grid resolution is near to the limit stated in (17); i.e. $r=0.5$. Table 4 shows that the convergence rates for CR-ML-P and CG-ML-P have decreased, but still remain stable for all refinement levels. The stand-alone multilevel solver did not convergence at all.

\begin{tabular}{|c|r|rrr|rrr|}
\hline \multirow{2}{*}{$\begin{array}{c}\text { Ref. } \\
\text { Depth }\end{array}$} & \multirow{2}{*}{ Nodes } & \multicolumn{3}{|c|}{ \#Iter } & \multicolumn{3}{|c|}{ CPU [sec] } \\
& & CR-ML-P & CG-ML-P & ML-P & CR-ML-P & CG-ML-P & ML-P \\
\hline 1 & 3180 & 27 & 26 & $\dagger$ & 8 & 8 & $\dagger$ \\
2 & 8010 & 27 & 27 & & 27 & 26 & \\
3 & 26887 & 28 & 28 & 121 & 116 & \\
4 & 64937 & 28 & 28 & 356 & 342 & \\
5 & 155951 & 31 & 31 & & 1171 & 1136 & \\
\hline
\end{tabular}

Table 4: Convergence history for $f=3 \mathrm{GHz}, r=0.49$. The $\dagger$ indicates that the solver did not converge.

Astonishingly, covergence could also be obtained beyond the critical limit for $r$. We carried out runs for $f=6 \mathrm{GHz}$ yielding $r=0.98$ on the initial mesh. However, as 
diplayed in table 5, the convergence has suffered tremendously, although remaining relatively stable. Even more surprising is the observation that the performance of the CG-algorithm was clearly superior, although in this situation not all the negative eigenvalues are captured by the direct coarse grid solver. There seems to be no straightforward explanation for this behaviour; one reason might be that the (simpler) $\mathrm{CG}$-algorithm is less affected by rounding errors.

\begin{tabular}{|c|r|rrr|rrr|}
\hline \multirow{2}{*}{$\begin{array}{c}\text { Ref. } \\
\text { Depth }\end{array}$} & \multirow{2}{*}{ Nodes } & \multicolumn{3}{|c|}{ \#Iter } & \multicolumn{3}{c|}{ CPU [sec] } \\
& & CR-ML-P & CG-ML-P & ML-P & CR-ML-P & CG-ML-P & ML-P \\
\hline 1 & 1349 & 55 & 55 & $\dagger$ & 9 & 8 & $\dagger$ \\
2 & 3076 & 164 & 161 & 86 & 76 & \\
3 & 11709 & 431 & 391 & 1017 & 887 & \\
4 & 66350 & 416 & 398 & 7702 & 7041 & \\
5 & 203587 & 468 & 396 & 29364 & 24693 & \\
\hline
\end{tabular}

Table 5: Convergence history for $f=6 \mathrm{GHz}, r=0.98$. The $\dagger$ indicates that the solver did not converge.

No convergence could be observed when we raised the frequency to $9 \mathrm{GHz}$ (corresponding to $r=1.47$ ).

To sum up some of the above results, we depict the convergence rates for several runs in figure 6 . The convergence rate is defined by

$$
\rho=\left(\frac{\left\|r_{m}\right\|}{\left\|r_{0}\right\|}\right)^{\frac{1}{m}},
$$

where $\left\|r_{m}\right\|$ denotes the euclidian norm of the residual after the (final) $m$-th iteration. $\rho$ gives the average decrease of the residual norm in each iteration.

We also carried out calculations substituting a symmetric QMR-algorithm [17] for the CR-iteration. Symmetric QMR was designed especially for complex symmetric matrices like occuring in our case. The convergence rates did not differ significantly, but we had to restrict the tolerance for the inner iteration (i.e. the projection scheme) by several orders of magnitude to obtain stability. So we consider this solver less favourable compared to CG and CR.

\section{Conclusion}

We have developed a nested multilevel scheme for the solution of indefinite complex linear systems which arise when the time-harmonic Maxwell's equations are discretized with edge elements. The inner iteration, being introduced to remove modes lying in the nullspace of the double-curl operator, restores an optimal convergence rate for the outer multilevel solver. Here the basic idea is that the large discrete nullspace of of the curl-operator can be represented by the gradient space of Lagrangian finite element functions: discrete potentials can be found in a proper finite element space. Thus our 


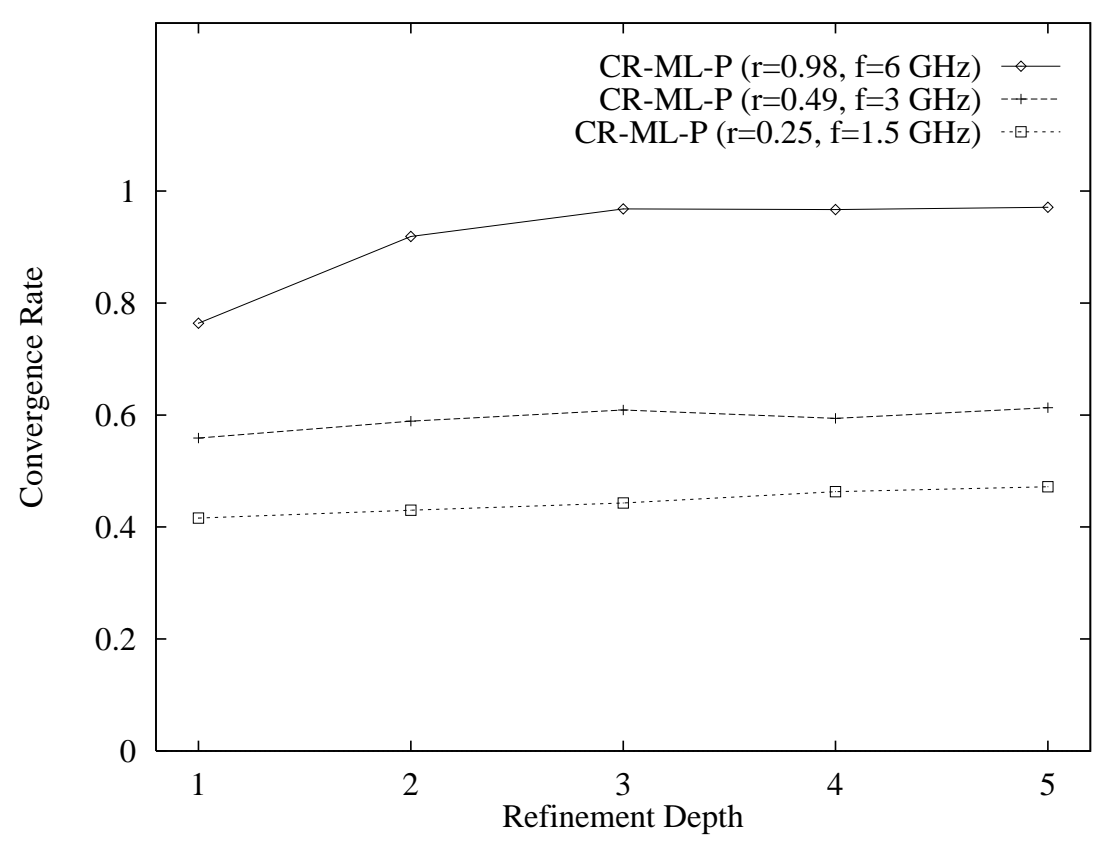

Figure 6: Convergence rates for different coarse grid resolutions.

multilevel approach also benefits from the close relationship between edge elements and differential forms, the very relationship that made them an attractive choice for the approximation of electric fields in the first place.

Numerical experiments revealed that - within the presented framework - the best performance can be achieved by employing the nested multilevel iteration as a preconditioner in a plain conjugate gradient iteration.

Further investigations could try to relax the restrictive requirements concerning the precision for the inner iteration. As pointed out in [23], an appealing alternative might arise from carrying out relaxation sweeps in parallel both in the Nédélec space and in the gradient space on each level of the multigrid iteration.

\section{References}

[1] M. Ainsworth, J. Z. Zhu, A. W. Craig, and O. C. Zienkiewicz. Analysis of the Zienkiewicz-Zhu a-posteriori error estimator in the finite element method. Int. J. $\mathrm{Nu}$ mer. Meths. in Eng., 28, pp. 2161-2174, 1989.

[2] F. Assous, P. Degond, P.A. Raviart, J. Segre, and E. Heintze. On a finite element method for solving the 3D Maxwell equations. J. Comp. Phys., 109:222-237, 1993.

[3] E. Baensch. Local mesh refinement in 2 and 3 dimensions. IMPACT Comput. Sci. Engrg., 3:181-191, 1991.

[4] D. Baldomir. Differential forms and electromagnetism in 3-dimensional Euclidean space $R^{3}$. IEE Proc. A, 133(3):139-143, 1986. 
[5] R. Bank and A. Weiser. Some a posteriori error estimators for elliptic partial differential equations. Math. Comp., 44:283-301, 1985.

[6] M. L. Barton and Z. J. Cendes. New vector finite elements for three-dimensional magnetic field computation. J. Appl. Phys., 61, pp. 3919-3921, 1987.

[7] R. Beck, B. Erdmann, and R. Roitzsch. KASKADE 3.0: An Object-Oriented Adaptive Finite Element Code. Technical report, Konrad-Zuse-Zentrum für Informationstechnik Berlin, 1995.

[8] R. Becker and R. Rannacher. Adaptive error control for multigrid finite element methods. Computing, 55(4):271-288, 1995.

[9] J. Bey. Tetrahedral grid refinement. Computing, 55(4):355-378, 1995.

[10] A. Bossavit. Whitney forms: a class of finite elements for three-dimensional computation in electromagnetism. Inst. Elec. Eng. Proc., Part A, 135, pp. 493-500(8), 1988.

[11] A. Bossavit. Solving Maxwell's equation in a closed cavity and the question of spurious modes. IEEE Trans. Magn., 26, pp. 702-705, 1990.

[12] F. Bossavit. A rationale for edge elements in 3D field computations. IEEE Trans. Mag., 24:74-79, 1988.

[13] J.H. Bramble. Multigrid methods. Pitman Research Notes in Mathematics Series. Longman, London, 1993.

[14] J.H. Bramble, D.Y. Kwak, and J.E. Pasciak. Uniform convergence of multigrid vcycle iterations for indefinite and nonsymmetric problems. SIAM J. Numer. Anal., 31(6):1746-1763, 1994.

[15] S. Caorsi and M. Raffetto. Electromagnetic boundary value problems in the presence of a partly lossy dielectric: considerations about the uniqueness of the solution. IEEE Trans. Microwave Theory Tech., 44(8, pp. 1511-1513), 1996.

[16] B.M. Dillon and J.P. Webb. A comparison of formulations for the vector finite element analysis of waveguides. IEEE Trans. Microwave Theory Tech., 42(2, pp. 308-316), 1994.

[17] Roland W. Freund. Conjugate gradient type methods for linear systems with complex symmetric coefficients. SIAM J. Sci. Stat. Comp., 13(1, pp. 425-448), 1992.

[18] N.A. Golias, A.G. Papagiannakis, and T.D. Tsiboukis. Efficient mode analysis with edge elements and 3-D adaptive refinement. IEEE Trans. Magn., 42(1, pp. 99-106), 1994.

[19] W. Hackbusch. Multi-grid Methods and Applications. Springer-Verlag, Berlin, 1985.

[20] Wolfgang Hackbusch. Iterative Solution of Large Sparse Linear Systems of Equations. Springer, Berlin, 1993.

[21] R. Hiptmair. Canonical construction of finite elements. Technical Report 360, Institut für Mathematik, Universität Augsburg, 1996.

[22] R. Hiptmair and M. Schemann. Multigrid method for Maxwell's equations. Technical Report in preparation, Math. Inst., Universität Augsburg, 1997. 
[23] Ralf Hiptmair. Multilevel Preconditioning for Mixed Problems in Three Dimensions. Ph.D. Thesis, Wissner, Augsburg, 1996.

[24] Jianming Jin. The Finite Element Method in Electrodynamics. Wiley, New York, 1993.

[25] C. Johnson and P. Hansbo. Adaptive finite element methods in computational mechanics. Comp. Meth. Appl. Mech. Eng., 101:143-181, 1992.

[26] Arnulf Kost. Numerische Methoden in der Berechnung elektromagnetischer Felder. Springer, Berlin, 1994.

[27] R. Leis. Initial boundary value problems in mathematical physics. B.G. Teubner, Stuttgart, 1986.

[28] N. Marcuwitz. Waveguide Handbook. P. Peregrinus, London, 1986.

[29] W. Mitchell. Unified multilevel adaptive finite element methods for elliptic problems. Technical Report UIUCDCS-R-88-1436, University of Illinois, 1988.

[30] P. Monk. A finite element methods for approximating the time-harmonic Maxwell equations. Numer. Math., 63:243-261, 1992.

[31] P. Monk and S. Zhang. Multigrid computation of vector potentials. J. Comp. Appl. Math., 62:301-320, 1995.

[32] J.C. Nédélec. Mixed finite elements in $\mathcal{R}^{3}$. Numer. Math., 35, pp. 315-341, 1980.

[33] K. D. Paulsen, Xilin Jia, and J. M. Sullivan. Finite element computations of specific absorption rates in anatomically conforming full-body models for hyperthermia treatment analysis. IEEE Trans. Biomed. Engrg., 40(9, pp. 933-945), 1993.

[34] S. Ramo, J. R. Whinnery, and Th. van Duzer. Fields and Waves in Communication Electronics. Wiley, New York, 1984.

[35] J.S. Savage and A.F. Peterson. High-order vector finite elements for tetrahedral cells. IEEE Trans Microwave Theory Tech., 44(6):874-879, 1996.

[36] P. P. Silvester and R. L. Ferrari. Finite Elements for Electrical Engineers. Cambridge University Press, Cambridge, 1990.

[37] H.A. van der Vorst and J.B.M Melissen. A Petrov-Galerkin type method for solving $A x=b$, where $A$ is symmetric complex. IEEE Trans. Magn., 26(2, pp. 706-708), 1990.

[38] R. Verführt. A Review of A Posteriori Error Estimation and Adaptive Mesh-Refinement Techniques. Wiley-Teubner, Chichester, 1996.

[39] H. Whitney. Geometric integration theory. Princeton Univ. Press, Princeton, 1957.

[40] H. Yserentant. Preconditioning indefinite discretization matrices. Num. Math., 54, pp. 719-734, 1988.

[41] O. C. Zienkiewicz and J. Z. Zhu. A simple error estimator and adaptive procedures for practical engineering analysis. Int. J. Numer. Meths. in Eng., 24, pp. 337-357, 1987. 